\title{
Continuous flow conditions for high temperature formation of a benzodioxan pharmaceutical intermediate: Rapid scaleup for early phase material delivery
}

\section{AUTHOR NAMES}

Pengbin Li, Shaohui Yang, ${ }^{i}$ Ruiheng Zhu,' Baoquan Sun, Zhongbo Li, ${ }^{i}$ Ping Huang, 'Jonas Y. Buser,ii Jose Miguel Minguez,iii Sarah J. Ryan ${ }^{\star i i}$

\section{AUTHOR ADDRESS}

i) Shanghai SynTheAll Pharmaceutical Co., Ltd. (“STA"), No. 7 Building, \#90 Delin Rd, WaiGaoQiao Free Trade Zone, Shanghai, 200131, People's Republic of China

ii) Eli Lilly and Company, Small Molecule Design and Development (SMDD), Lilly Technology Center North, 1223 W. Morris St, Indianapolis, IN, 46221

iii) Eli Lilly and Company, Discovery Chemistry Research and Technologies (DCRT), Avda. de la Industria 30, 28108 Alcobendas, Madrid, Spain. 


\section{Index}

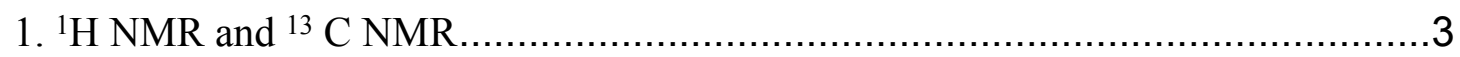

1) 1-(4-((3,4-dichlorobenzyl)oxy)phenyl)ethan-1-one (3) ........................

2) 2-Bromo-1(4-((3,4-dichlorobenzyl)oxy)phenyl)ethan-1-one (4) .................5

3) 3-(2-(4-((3,4-dichlorobenzyl)oxy)phenyl-2-oxoethoxy)-4-fluorobenzonitrile

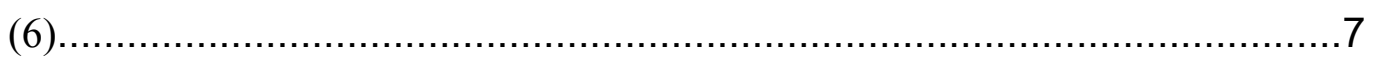

4) (S)-3-(2-(4-((3,4-dichlorobenzyl)oxy)-2-hydroxyethoxy)-4-fluorobenzonitrile

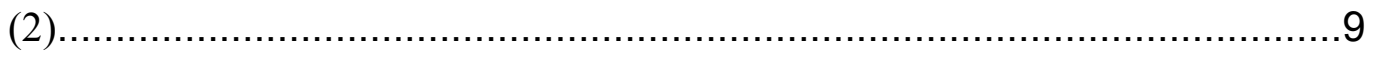

5) (S)-2-(4-((3,4-dichlorobenzyl)oxy)phenyl)-2,3-dihydrobenzo [b][1,4]dioxine-

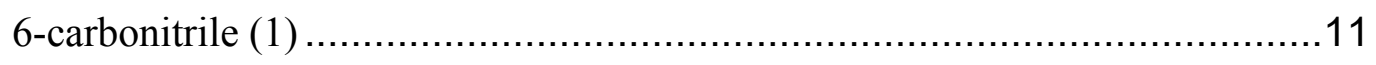

2. Chromatogram of the racemate and the enantiomerically pure compound 13

1) (S)-3-(2-(4-((3,4-dichlorobenzyl)oxy)-2-hydroxyethoxy)-4-fluorobenzonitrile

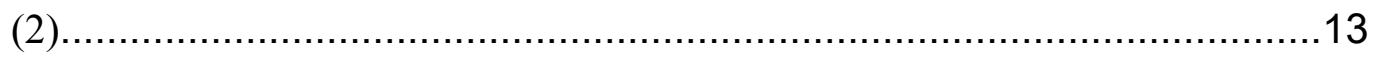

2) (S)-2-(4-((3,4-dichlorobenzyl)oxy)phenyl)-2,3-dihydrobenzo [b][1,4]dioxine-

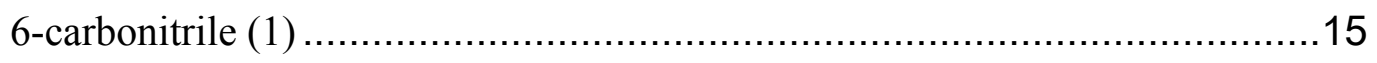

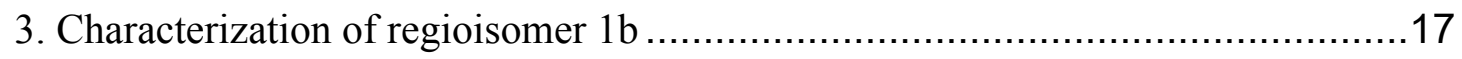

4. Safety Analysis of the high temperature $\mathrm{S}_{\mathrm{N}} \mathrm{Ar}$ Cyclization Conditions ...............23 
5. Port Connector reactors for Small Scale Screening of High Temperature Conditions .24 


\section{1. ${ }^{1} \mathrm{H}$ NMR and ${ }^{13}$ C NMR}

1) 1-(4-((3,4-dichlorobenzyl)oxy)phenyl)ethan-1-one (3)

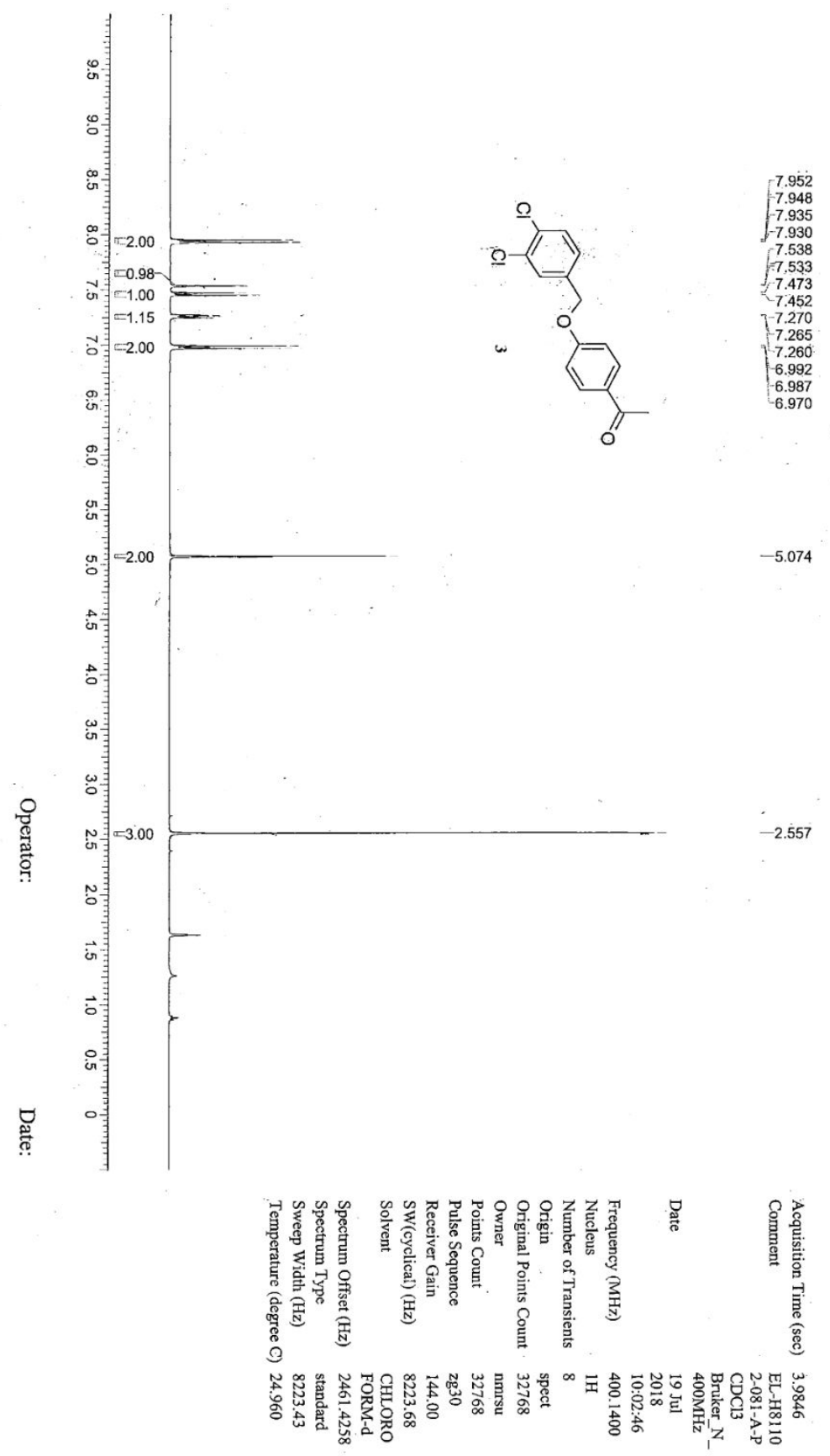




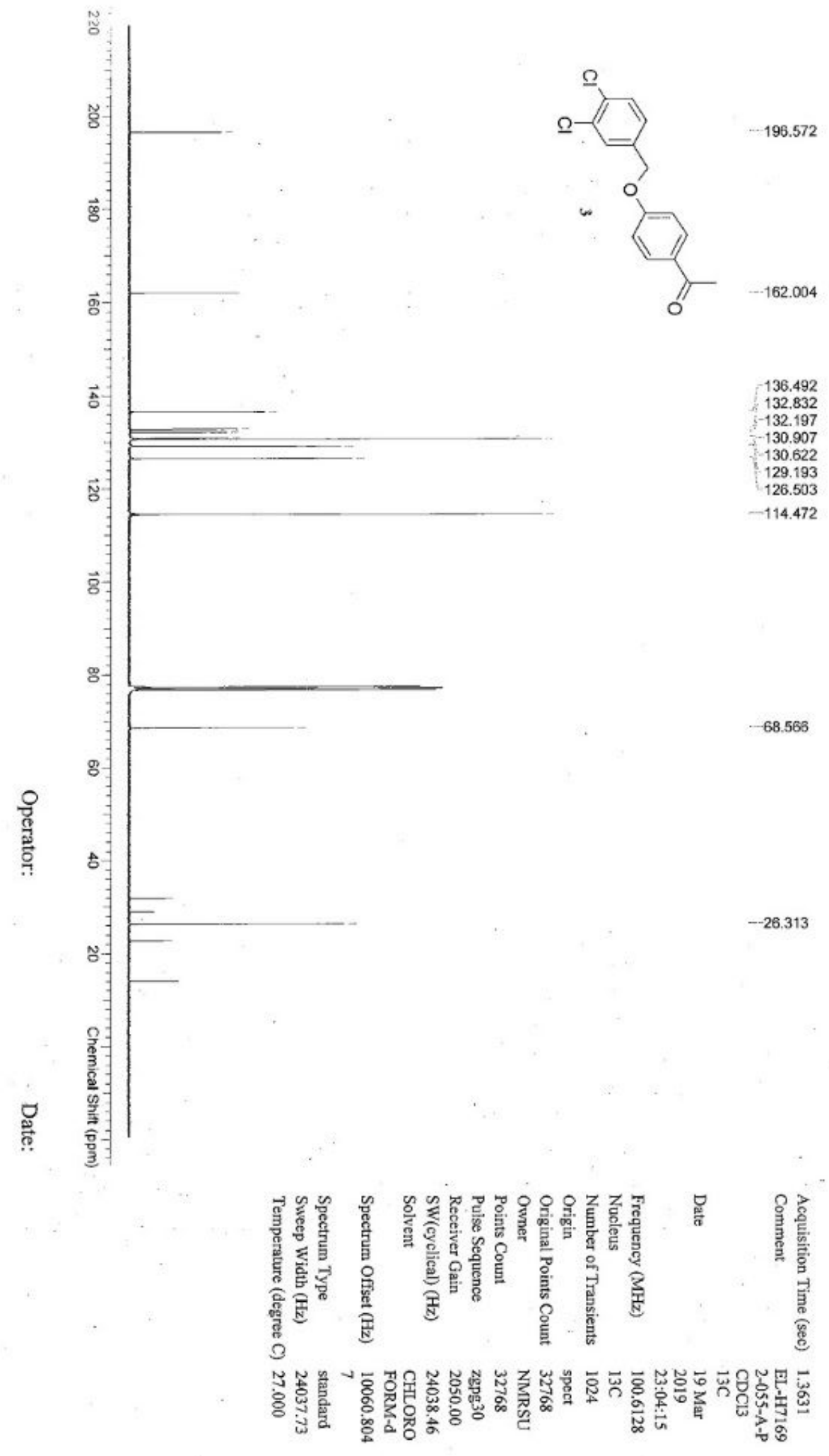


2) 2-Bromo-1(4-((3,4-dichlorobenzyl)oxy)phenyl)ethan-1-one (4)

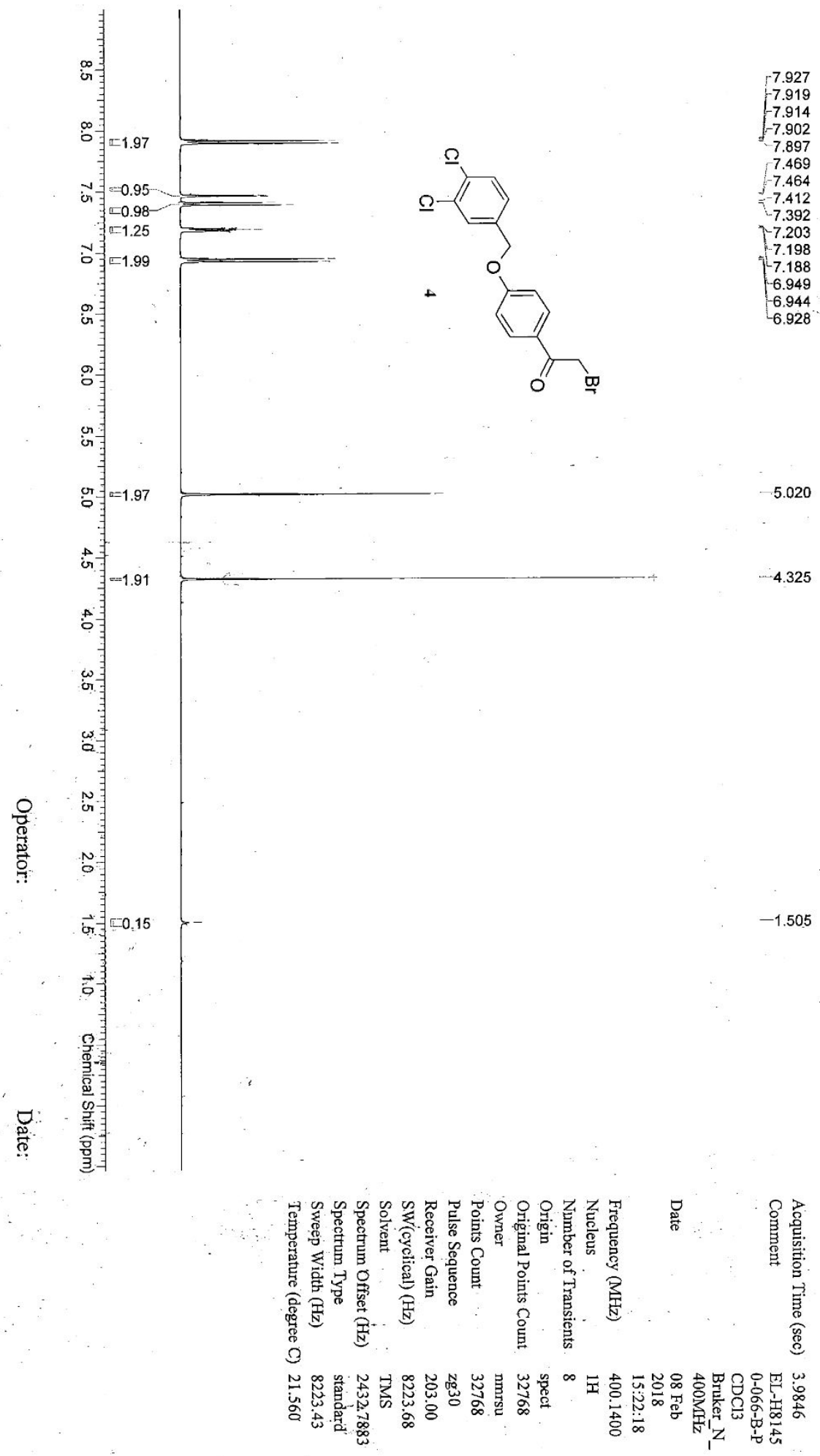




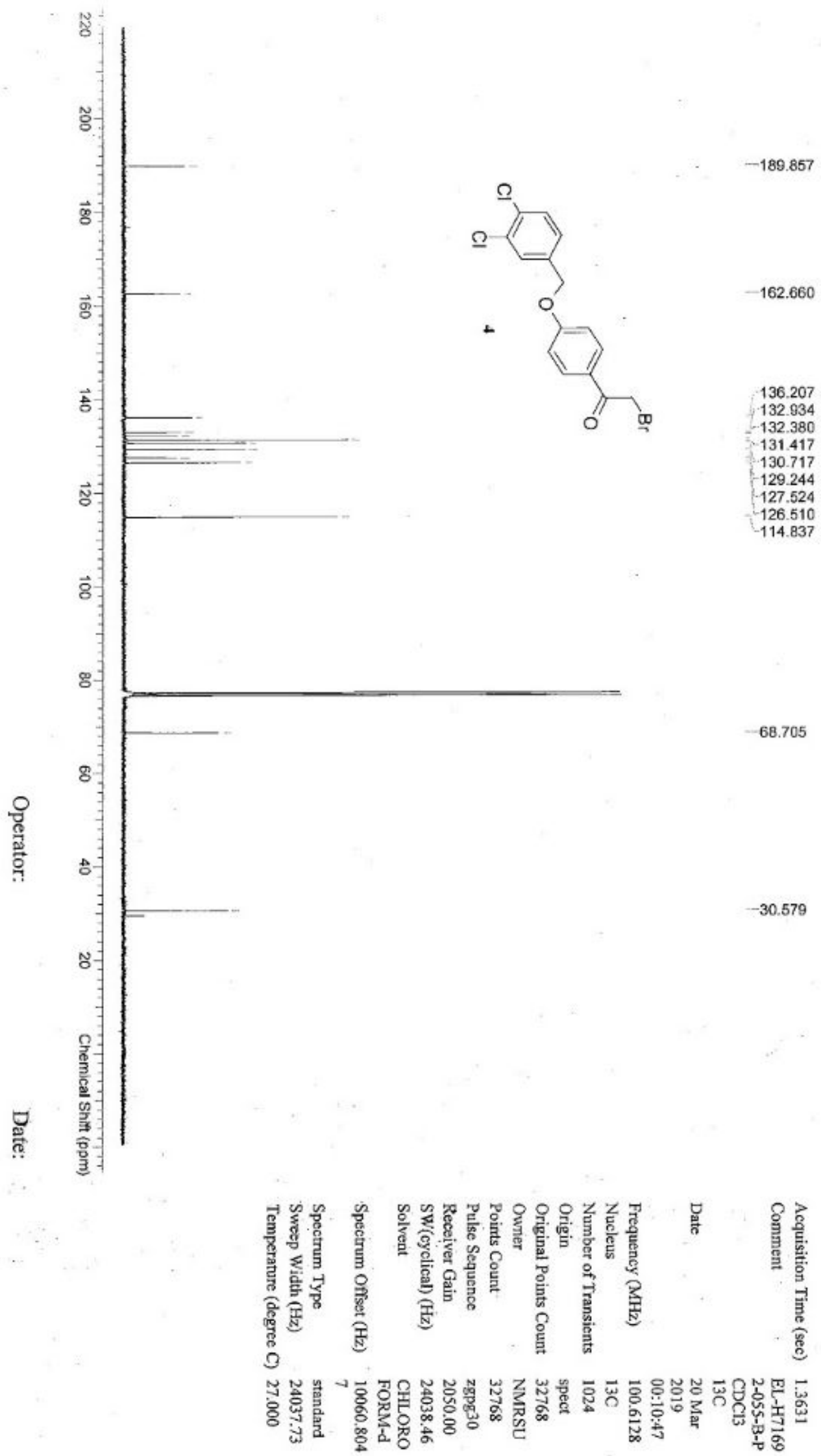


3) 3-(2-(4-((3,4-dichlorobenzyl)oxy)phenyl-2-oxoethoxy)-4-

fluorobenzonitrile (6)

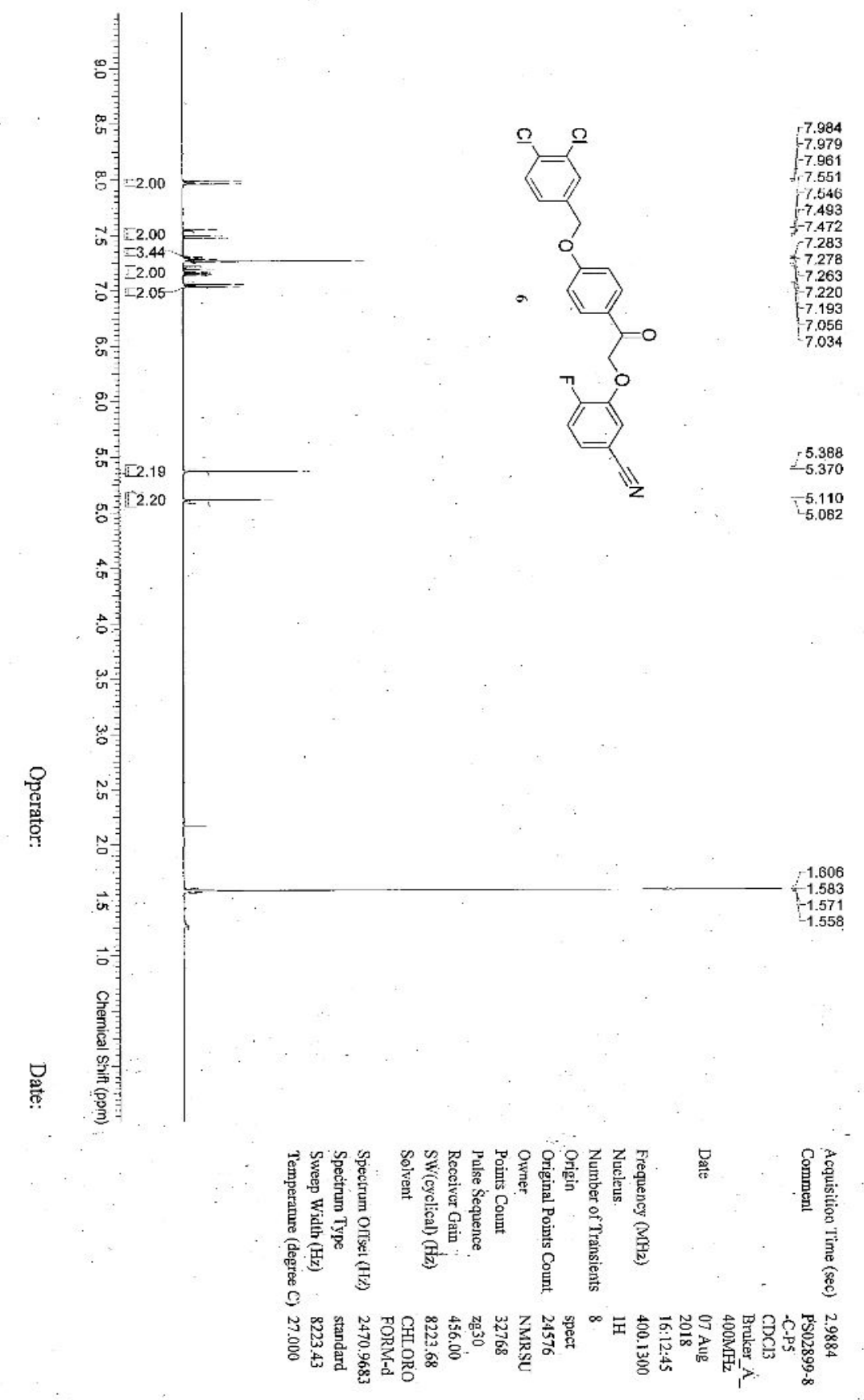



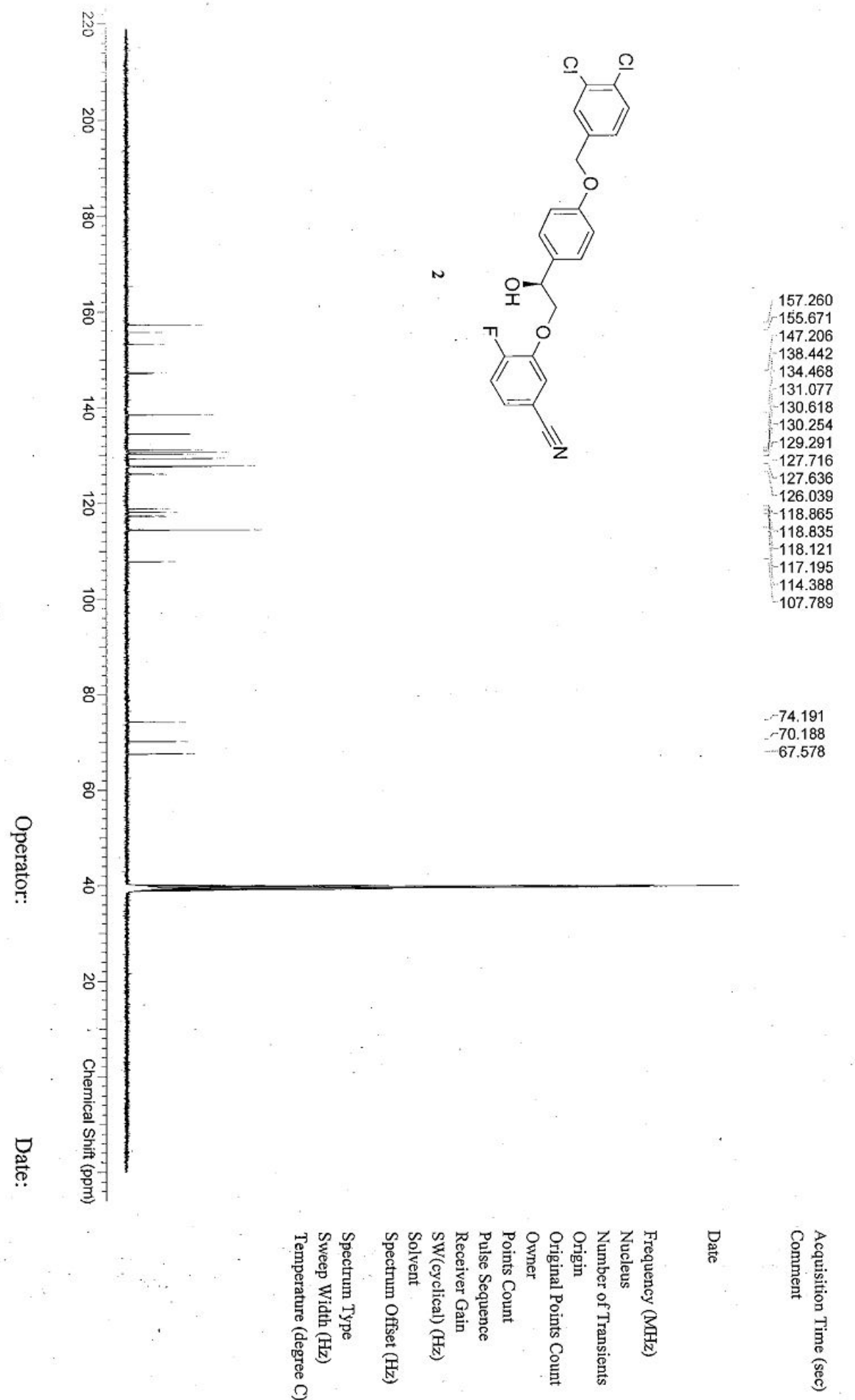

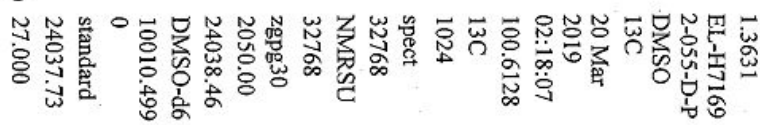


4) (S)-3-(2-(4-((3,4-dichlorobenzyl)oxy)-2-hydroxyethoxy)-4-

fluorobenzonitrile (2)

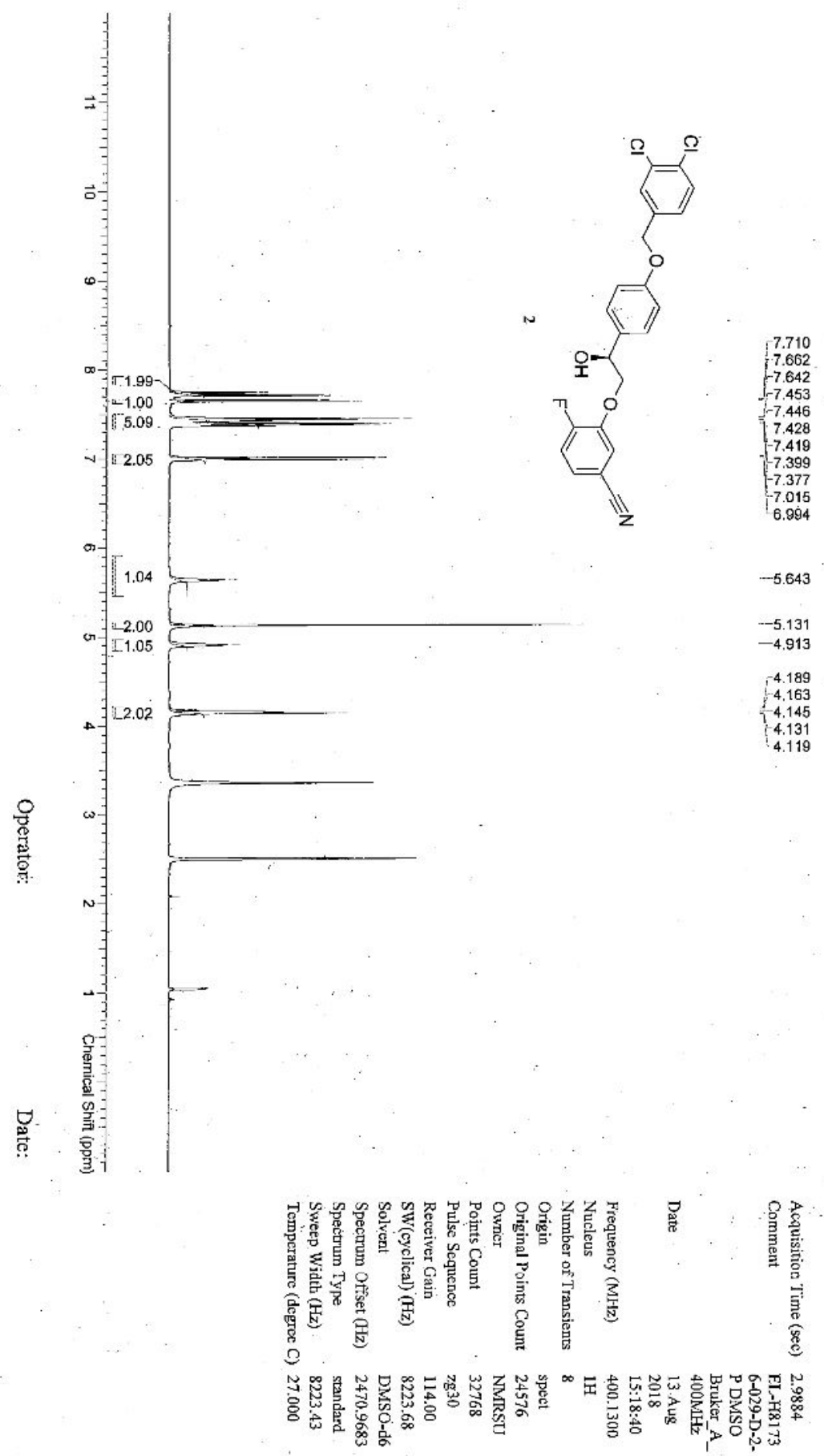




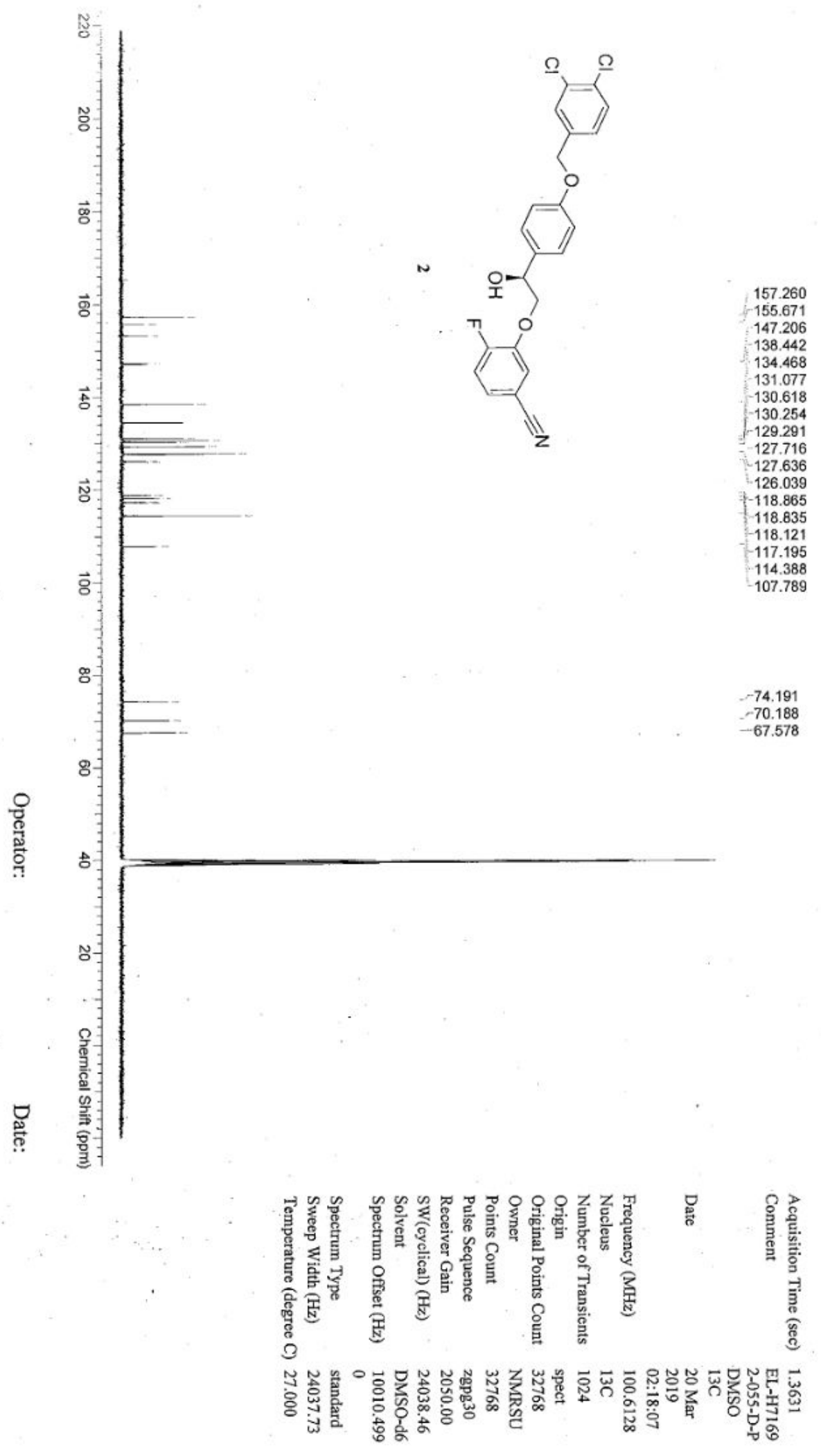


5) (S)-2-(4-((3,4-dichlorobenzyl)oxy)phenyl)-2,3-dihydrobenzo

[b][1,4]dioxine-6-carbonitrile (1)

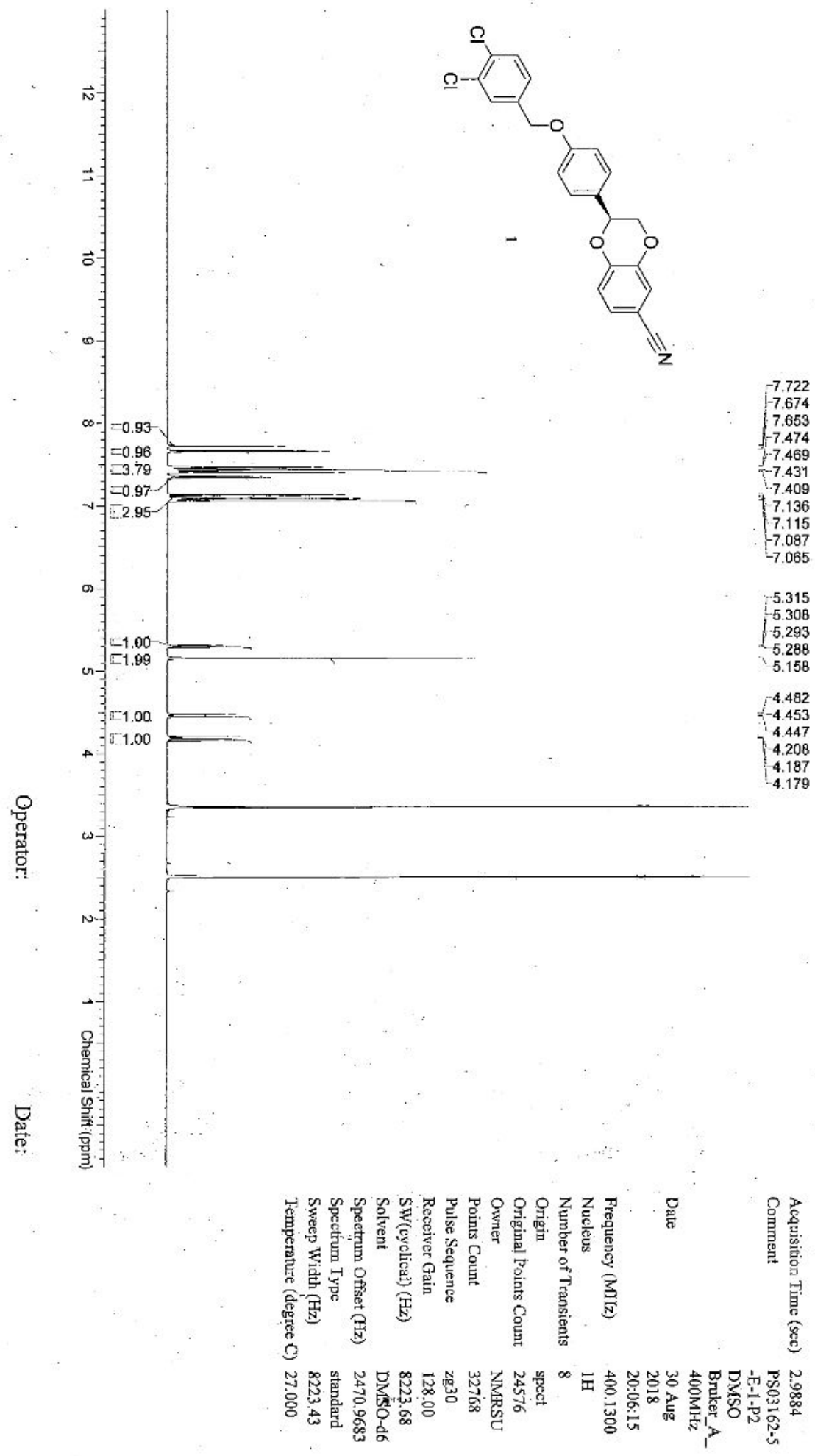




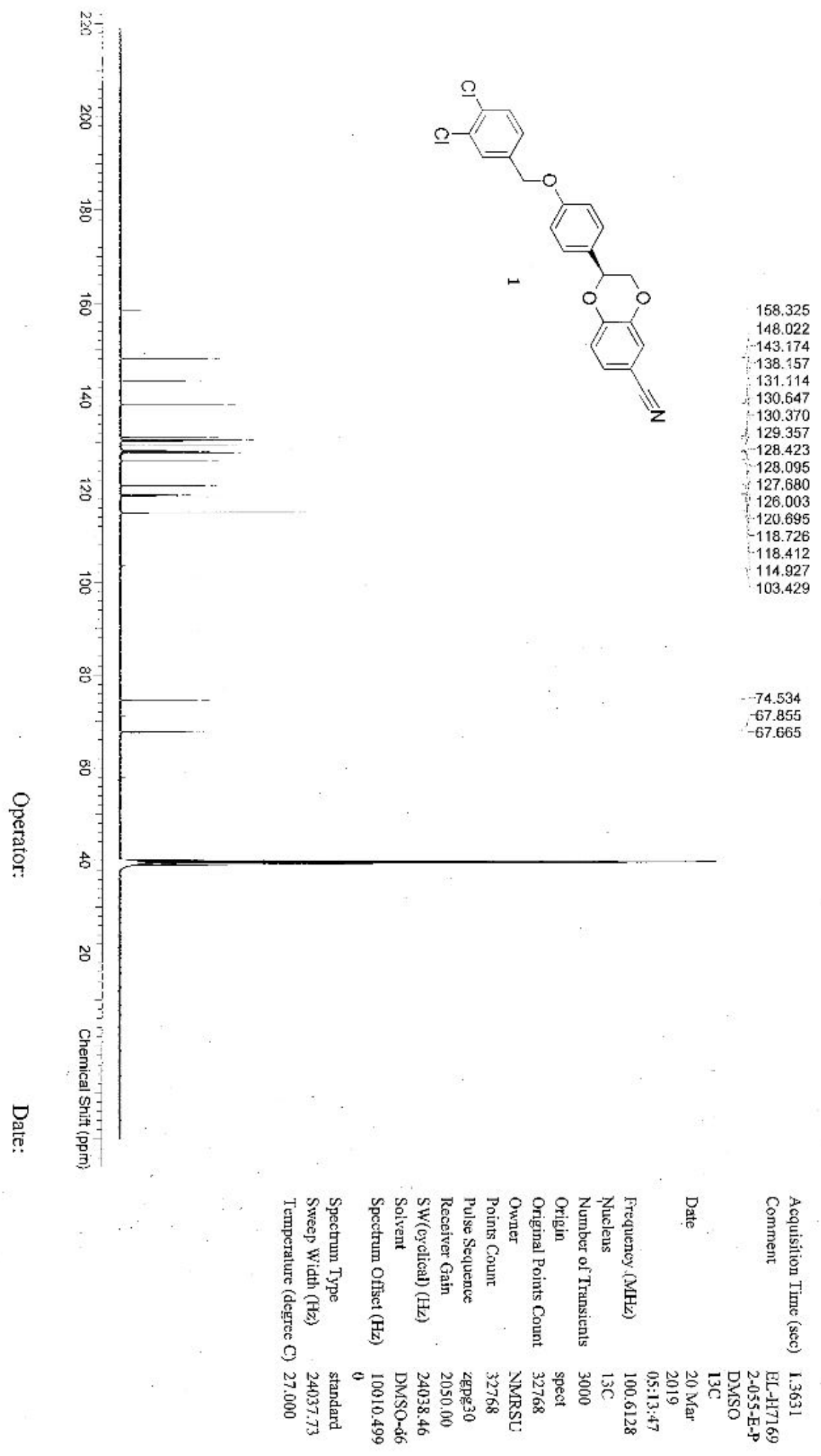




\title{
2. Chromatogram of the racemate and the enantiomerically
}

\author{
pure compound
}

\section{1) (S)-3-(2-(4-((3,4-dichlorobenzyl)oxy)-2-hydroxyethoxy)-4-}

\section{fluorobenzonitrile (2)}

\section{HPLC REPORT}
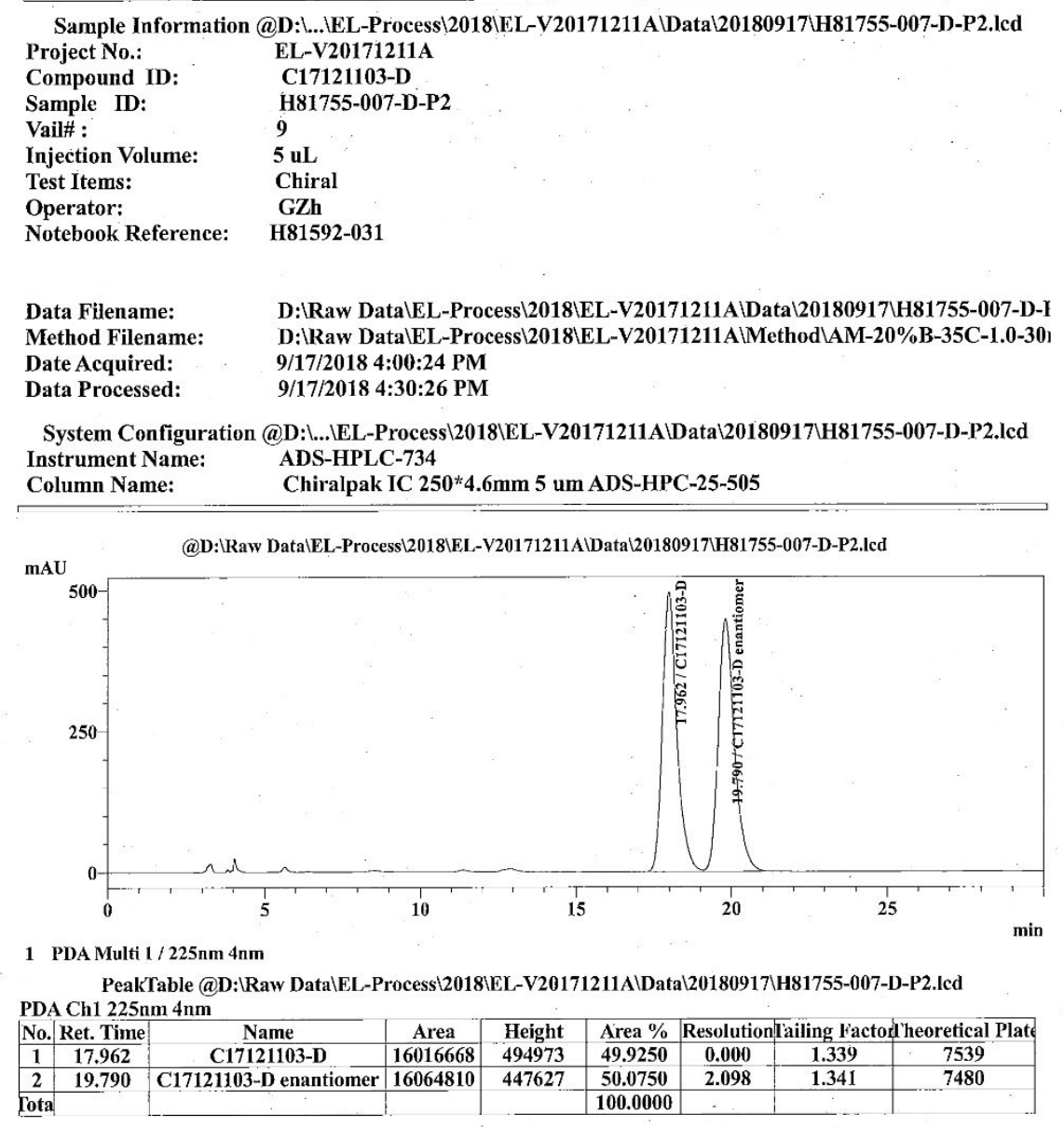


\section{HPLC REPORT}

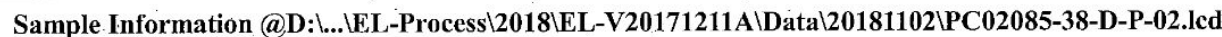
Project No. Compound ID: Sample ID:

Vail\# : EL-V20171211A

Injection Volume:

Test Items:

C17121103-D

Operator:

PC02085-38-D-P

Notebook Reference:

Data Filename:

Method Filename:

Date Acquired:

10

$10 \mathrm{uL}$

Chira

GZh

H81592-050

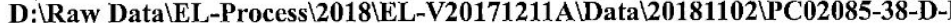
D:\Raw DatalEL-Process $\mid 2018 \backslash$ EL-V20171211A $\backslash$ Method $\backslash A M-20 \% B-35 C-1.0-30$ । 11/2/2018 6:45:09 PM

Data Processed: $\quad$ 11/5/2018 9:33:58 AM

System Configuration @.D:i...LEL-ProcessL2018\EL-V20171211A|Datai20181102\PC02085-38-D-P-02.led Instrument Name: ADS-HPLC-734

Column Name:

Chiralpak IC 250*4.6mm 5 um ADS-HPC-25-505

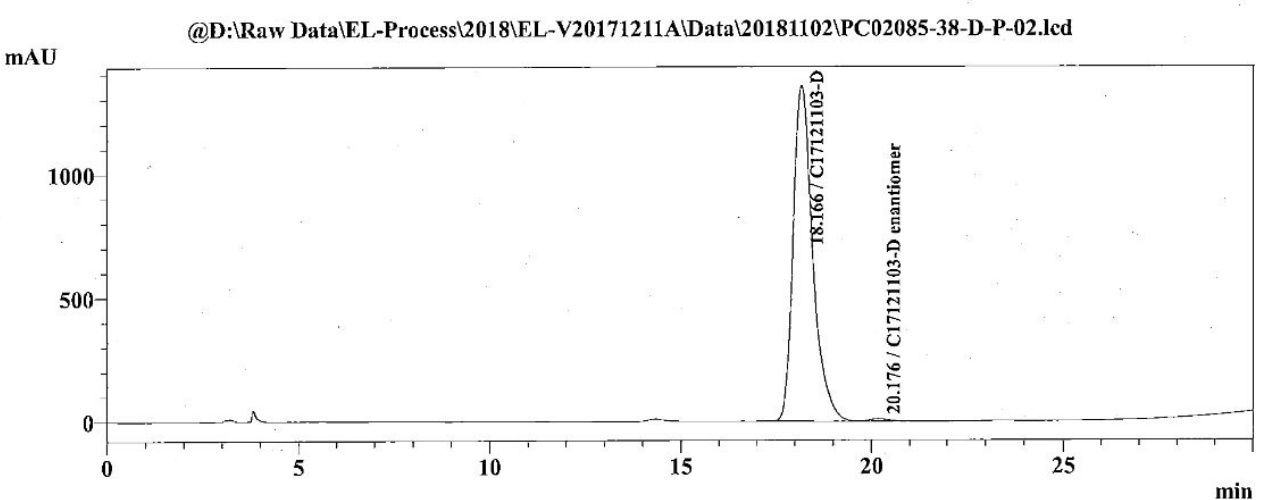

1 PDA Multi 1 / 225nm 4nm

PeakTable @D: |Raw DatalEL-Process\2018\EL-V20171211A|Data|20181102\PC02085-38-D-P-02.lcd

PDA Ch1 225nm 4nm

\begin{tabular}{|c|c|c|c|c|c|c|c|c|}
\hline Peak\# & Ret. Time & Name & Area & Height & Area \% & Resolution Tailing Facton Theoretical Plate \\
\hline 1 & 18.166 & C17121103-D & 48555041 & 1356244 & $\mathbf{9 9 . 2 9 8 3}$ & 0.000 & 1.399 & 6554 \\
\hline 2 & 20.176 & C17121103-D enantiome & 343115 & $\mathbf{9 1 5 3}$ & $\mathbf{0 . 7 0 1 7}$ & 2.178 & $\mathbf{0 . 0 0 0}$ & $\mathbf{7 2 4 9}$ \\
\hline Total & & & & 100.0000 & & & \\
\hline
\end{tabular}




\section{2) (S)-2-(4-((3,4-dichlorobenzyl)oxy)phenyl)-2,3-dihydrobenzo}

\section{[b][1,4]dioxine-6-carbonitrile (1)}

\section{HPLC REPORT}
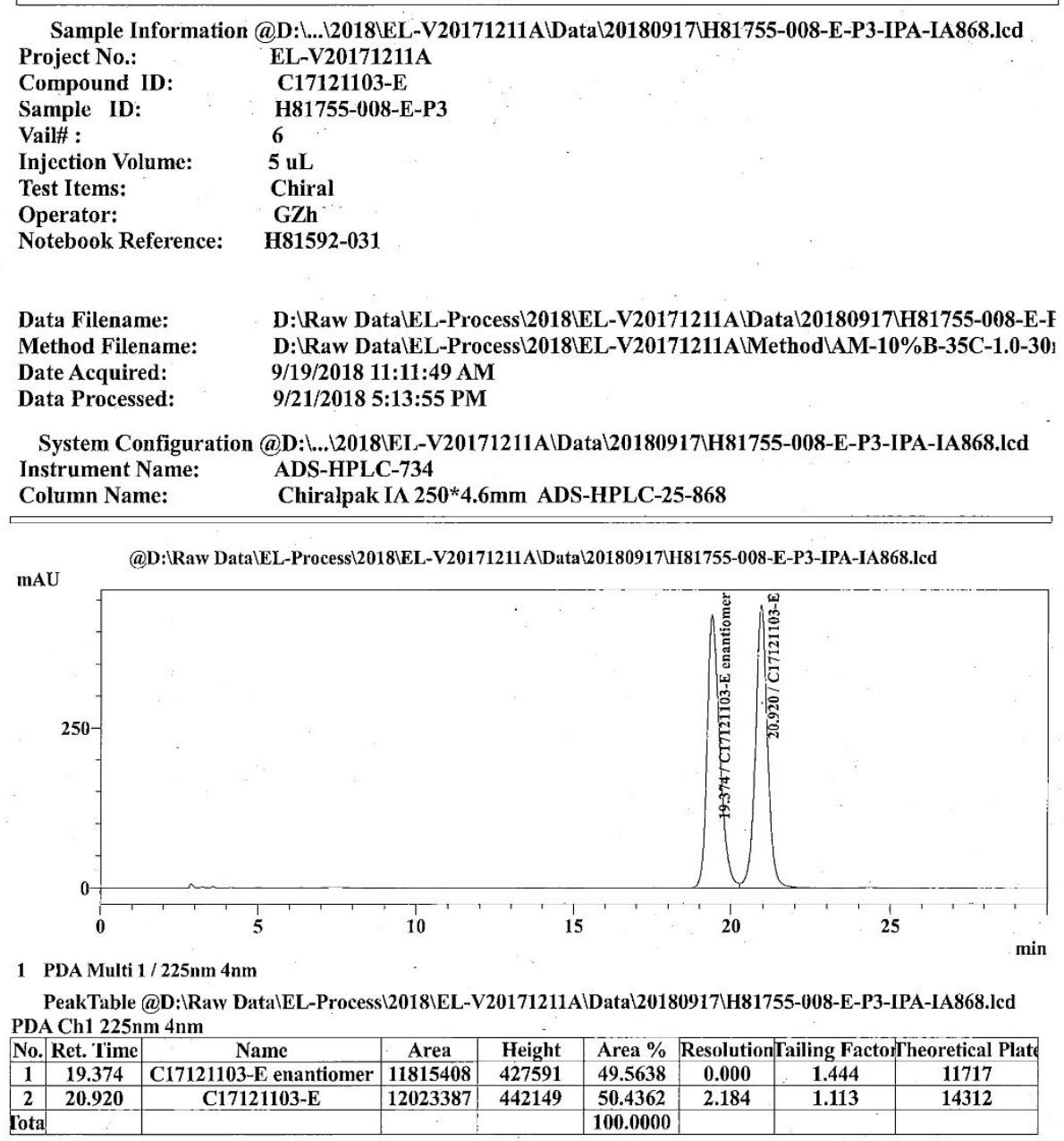


\section{HPLC REPORT}

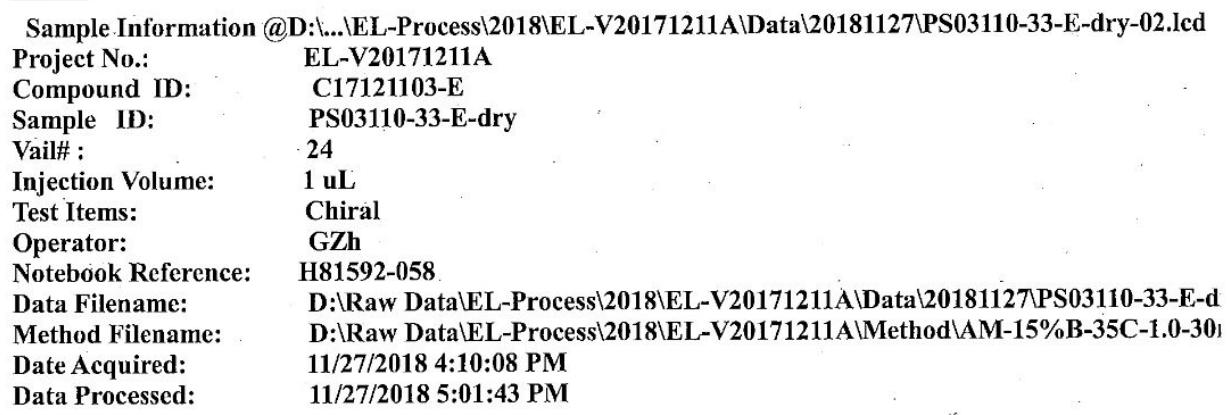

Sample.Information @ @D: \....EL-Process $\backslash 2018 \backslash E L-V 20171211 A \backslash D a t a \mid 20181127 \backslash P S 03110-33-E-d r y-02 . I c d$ Project No.: EL-V20171211A

Compound ID: C17121103-E

Sample ID: PS03110-33-E-dry

Vail\# :

Injection Volume: $\quad 1 \mathrm{uL}$

Test Items: Chiral

Operator:

GZh

Notebook Reference: H81592-058

Data Filename:

Method Filename:

D: \Raw DataLEL-Process $\backslash 2018 \backslash$ EL-V20171211A $\mid$ Data $\backslash 20181127>P S 03110-33-E-d$

Date Acquired: D: \Raw Data $L E L-P r o c e s s \backslash 2018 \backslash E L-V 20171211 A \backslash M$ Method $\backslash A M-15 \% B-35 C-1.0-30\rfloor$

Data Processed:

11/27/2018 4:10:08 PM 11/27/2018 5:01:43 PM

System Configuration @D: $1 . . .12018 \backslash$ EL-V20171211ADDatal20181127\PS03110-33-E-dry-02.lcd Instrument Name: ADS-HPLC-734

$\mathrm{mAU}$

@D:|Raw DatalEL-Process\2018\EL-V20171211A\DataL20181127\PS03110-33-E-dry-02.Icd

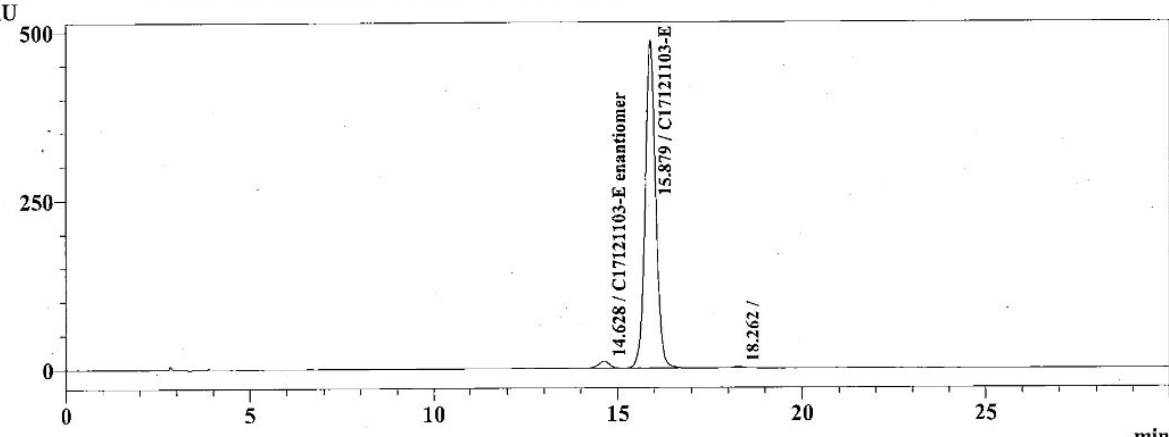

1 PDA Multi $1 / 225 \mathrm{~nm} 4 \mathrm{~nm}$

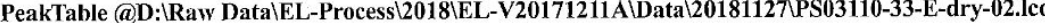
PDA Ch1 225nm 4nm

\begin{tabular}{|c|c|c|c|c|c|c|c|c|}
\hline Peak\# & Ret. Time & Name & Area & Height & Area \% & \multicolumn{3}{|c|}{ ResolutionTailing Factor Theoretical Plate } \\
\hline 1 & 14.628 & C17121103-E enantiome & 220600 & 10331 & 2.1845 & 0.000 & 0.927 & 11082 \\
\hline 2 & 15.879 & C17121103-E & 9825908 & 485836 & 97.2999 & 2.308 & 1.057 & 14496 \\
\hline 3 & 18.262 & & 52070 & 2463 & 0.5156 & 4.335 & 1.084 & 16305 \\
\hline Total & & & & & 100.0000 & & & \\
\hline
\end{tabular}




\section{Characterization of regioisomer $1 b$}

The regioisomers 1 and $1 \mathrm{~b}$ could not be separated by flash column chromatography. Identification of the product regioisomer $1 \mathrm{~b}$ was achieved by product derivatization. The mixture of 1 and $1 \mathrm{~b}$ were converted to the aldehyde via treatment with DIBAL-H according to the procedure below. The isomers were separated by flash column chromatography and analyzed by $2 \mathrm{D}$ NMR spectroscopy to confirm the regioisomer $1 \mathrm{~b}$.

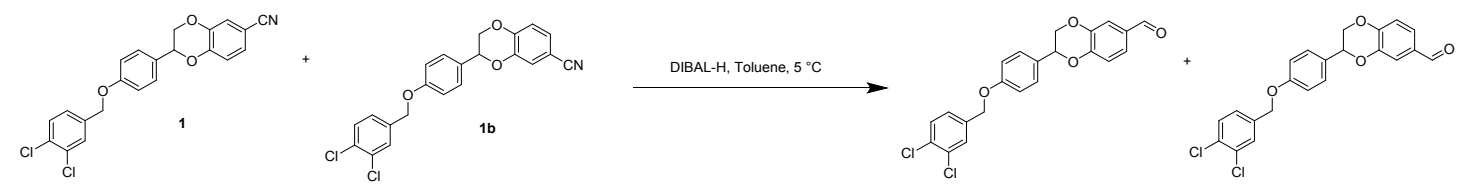

To a $100 \mathrm{~mL}$ 3-necked RBF was added the mixture of 1 and $1 \mathrm{~b}(3.6 \mathrm{~g}, 8.7$ mmol, 1.0 eq.), toluene ( $28 \mathrm{~mL}, 8 \mathrm{~mL} / \mathrm{g}$ ). After the suspension was cooled to 5 ${ }^{\circ} \mathrm{C}$, DIBAL-H (8 mL, $150 \mathrm{mmol}, 1.5$ eq., in $1 \mathrm{M}$ toluene solution) was added into the reaction mixture dropwise. The reaction mixture was stirred for $2 \mathrm{~h}$ at $5^{\circ} \mathrm{C}$. After which time, it was quenched with potassium tartrate $(25 \mathrm{~g}, 20 \mathrm{wt} \%$, aq.) and stirred at RT for $1 \mathrm{~h}$. The solid was removed by filtration through a pad of celite, then the organic layer was separated and washed with $2 \mathrm{M} \mathrm{HCl}$. The volatiles were removed, and the residue was purified via column chromatography to separate the two product regioisomers. 
Desired Regioisomer 


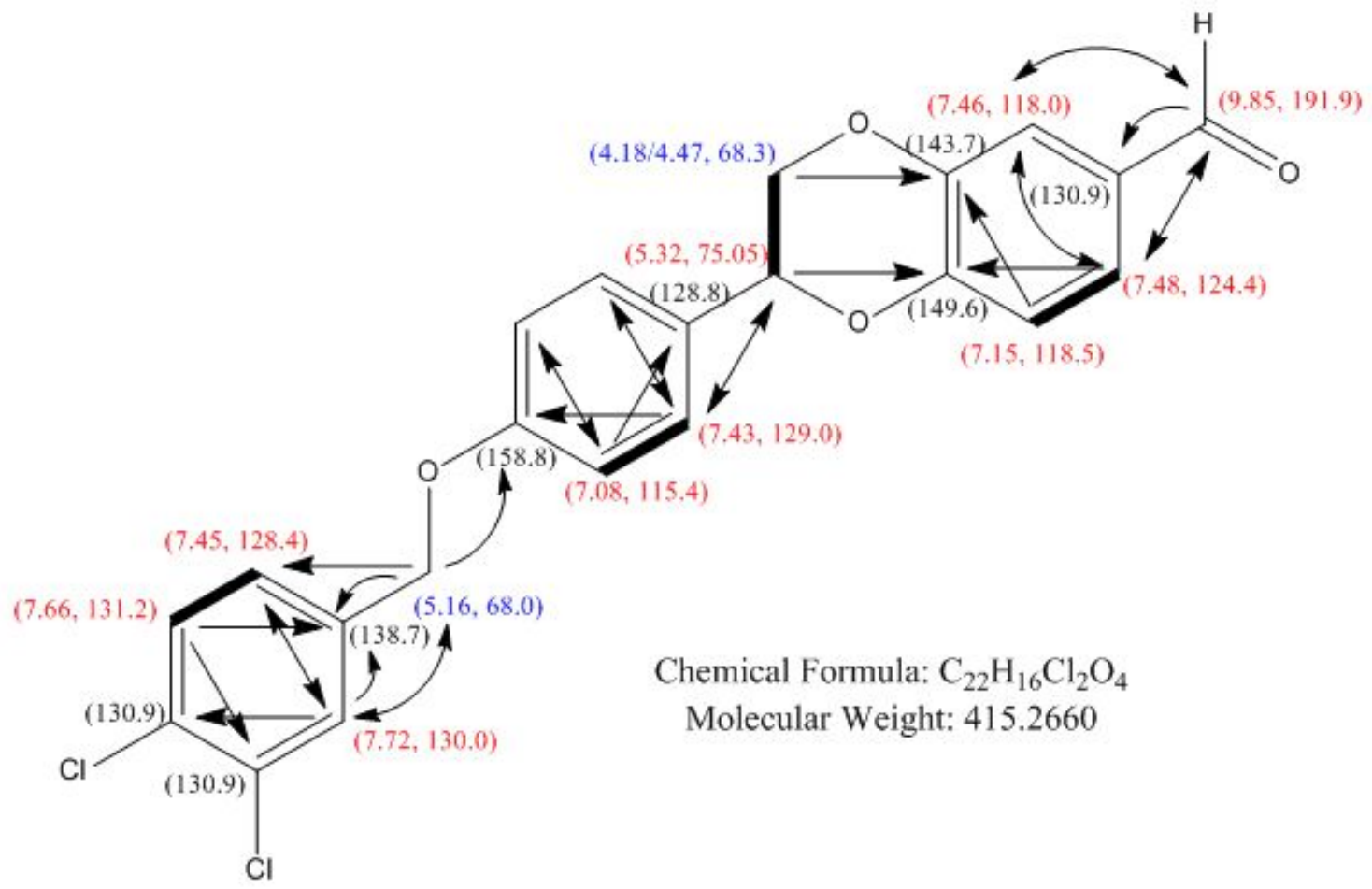

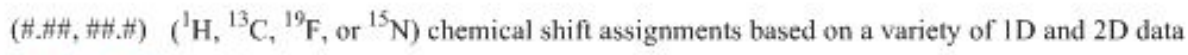

$\left(H . H H\right.$, thit.H) $\left({ }^{1} \mathrm{H},{ }^{13} \mathrm{C}\right)$ chemical shift assignments of positive correlation in $\mathrm{HSQC}$ (with multiplicity editing) $\mathrm{CH}_{\text {or }} \mathrm{CH}_{3}$ $(\# . \#,+\#)\left({ }^{1} \mathrm{H},{ }^{13} \mathrm{C}\right.$ ) chemical shift assignments of negative correlation in $\mathrm{HSQC}$ (with multiplicity editing) $\mathrm{CH}_{2}$

Represents an HMBC long range correlation (LRC) was observed $\left({ }^{1} \mathrm{H}\right.$ to ${ }^{13} \mathrm{C}$ ) or $\left({ }^{1} \mathrm{H}\right.$ to ${ }^{15} \mathrm{~N}$ )

Represents an COSY correlation was observed between proton resonances

1H

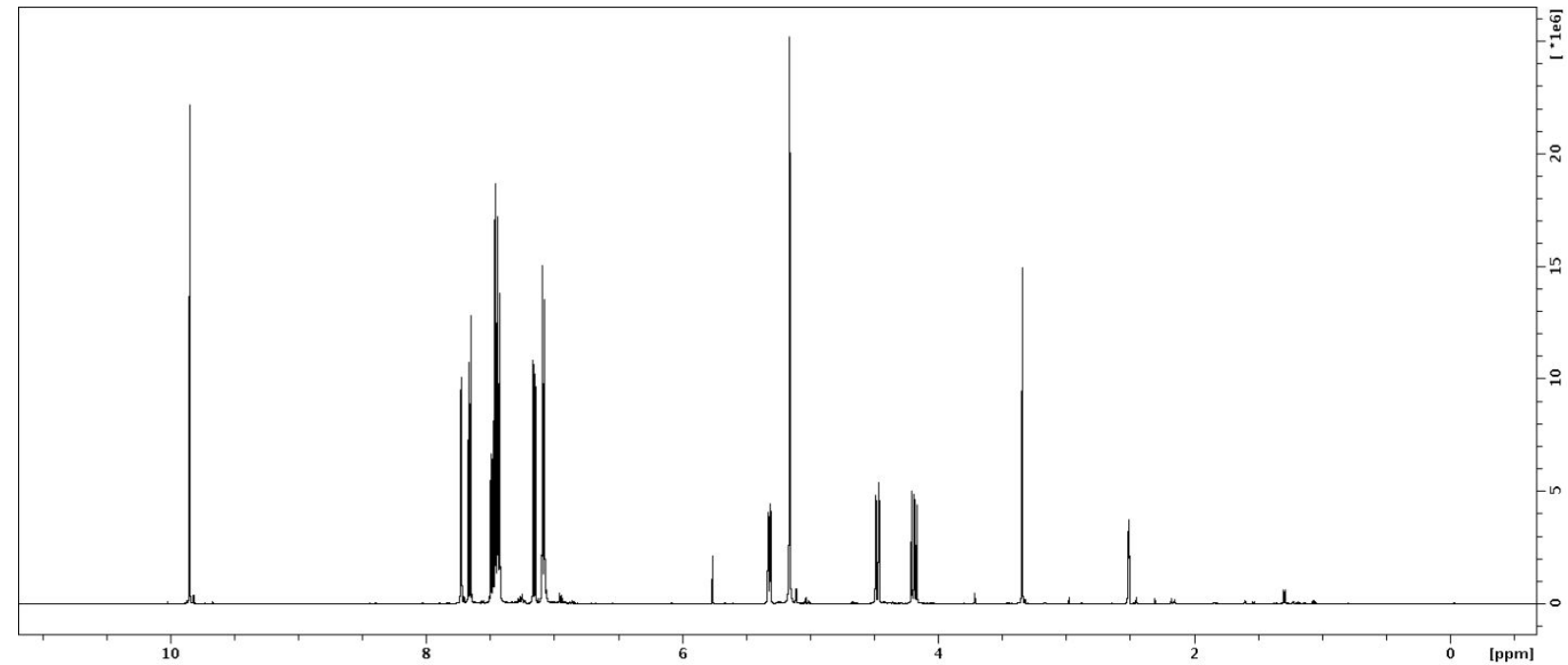


HSQC

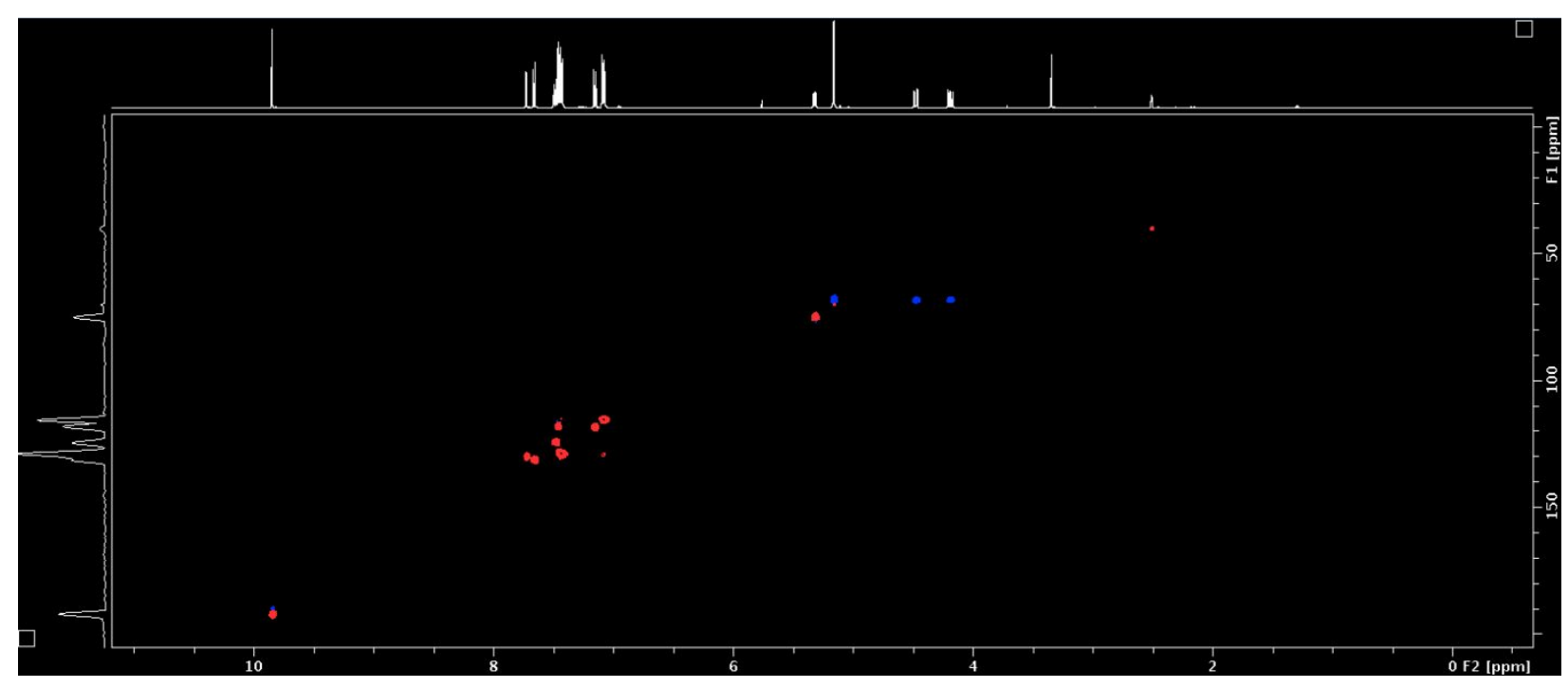

HMBC

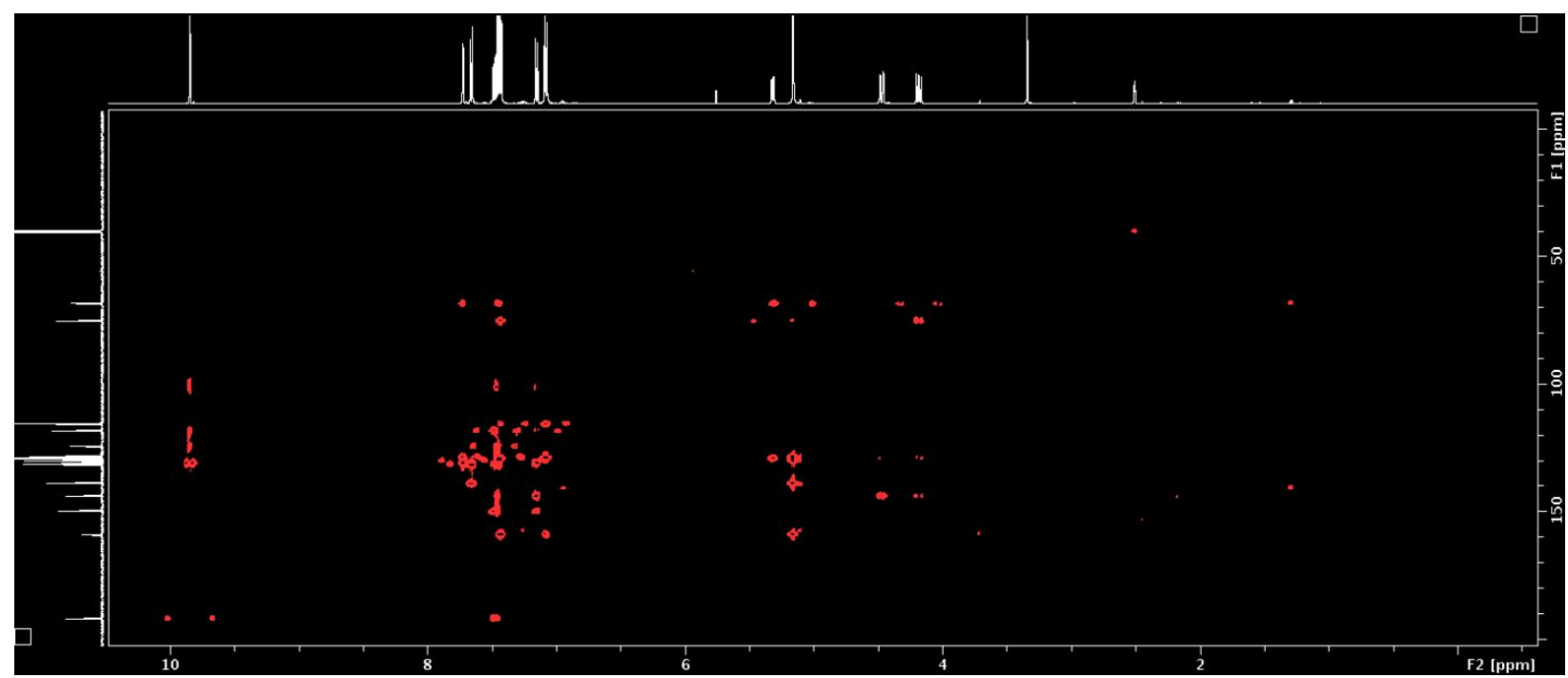


COSY

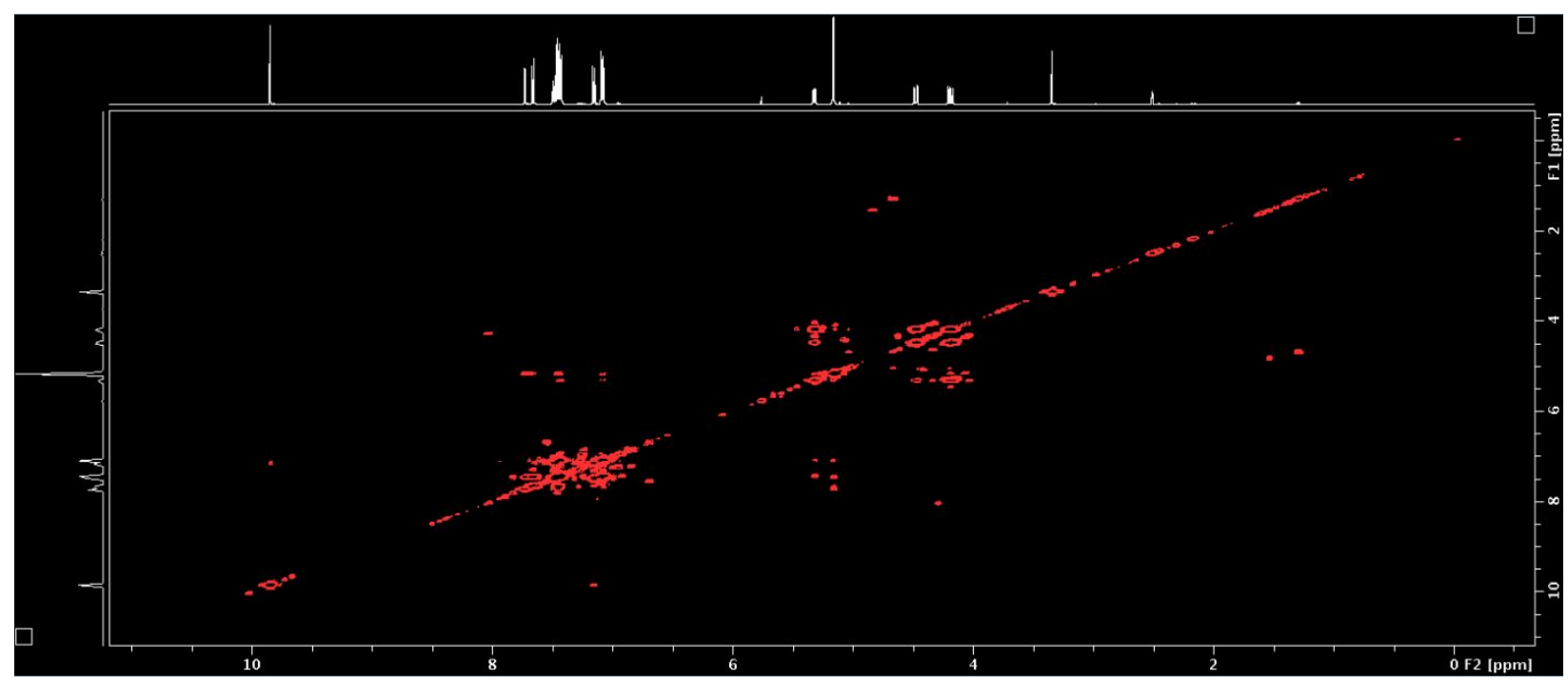

CARBON 


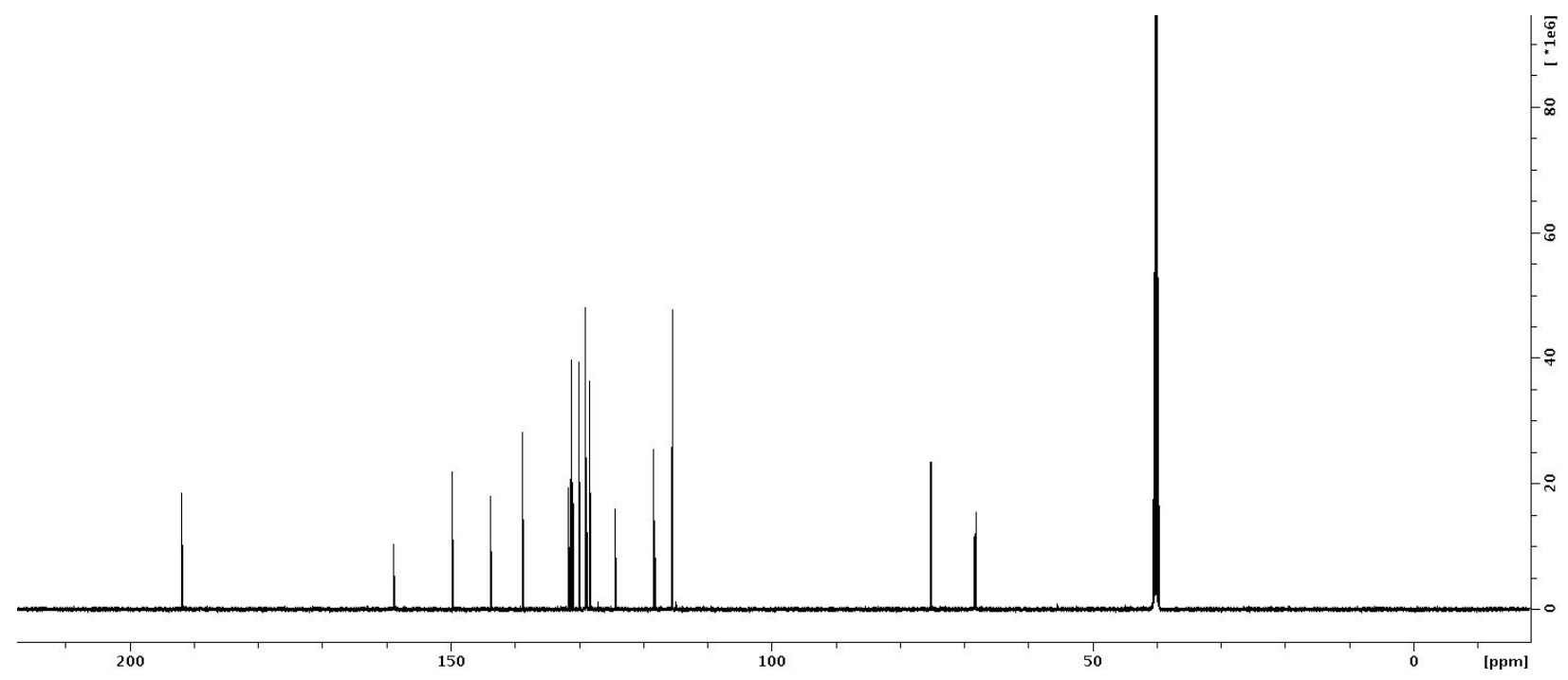

Undesired Regioisomer 


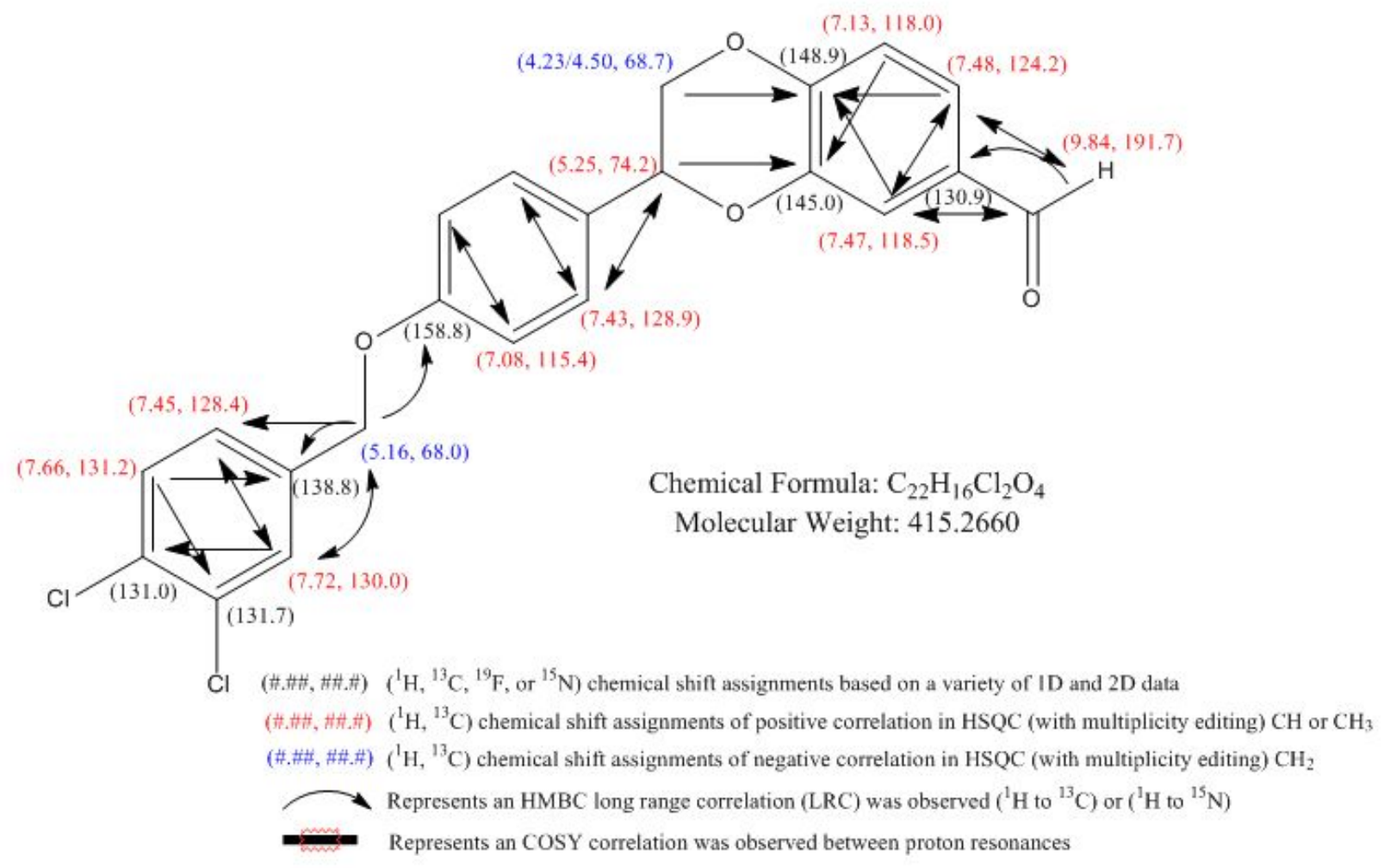

$1 \mathrm{H}$

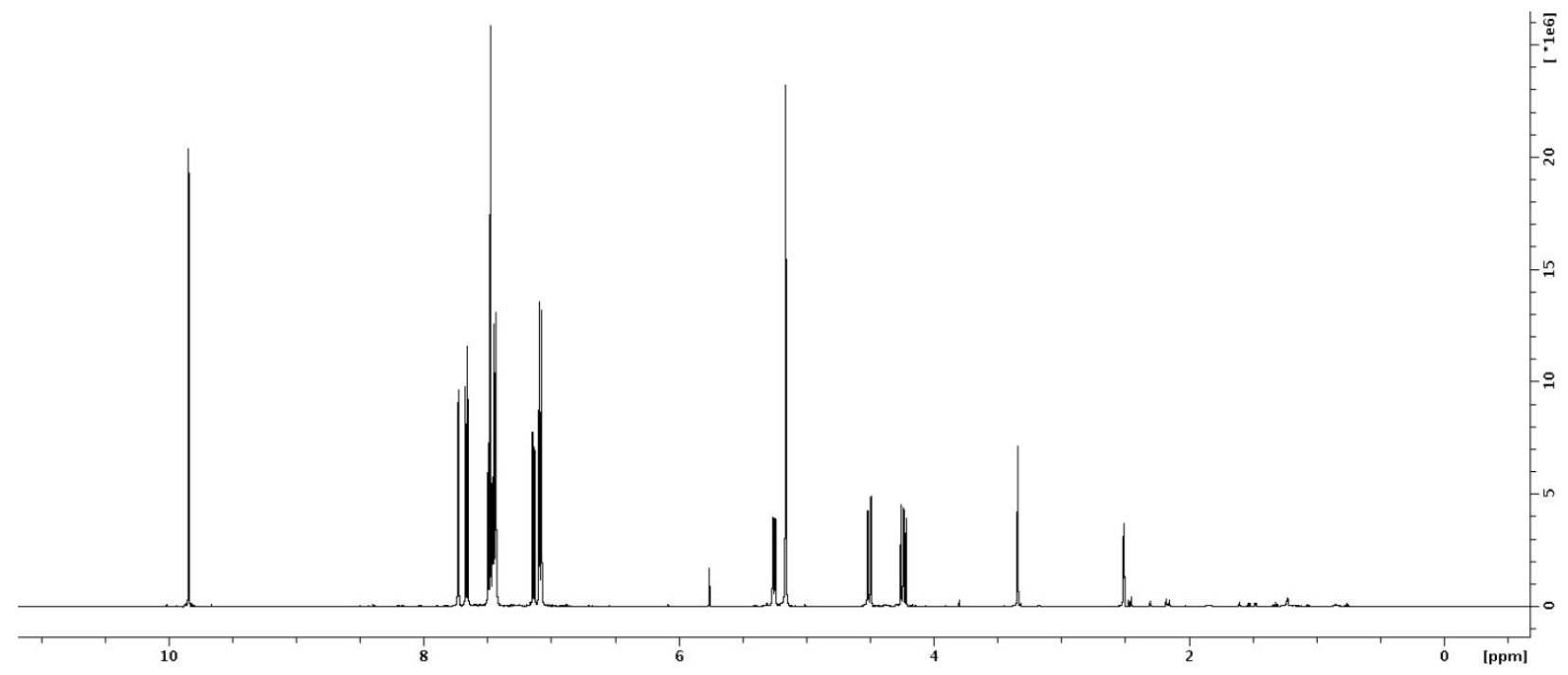


HSQC

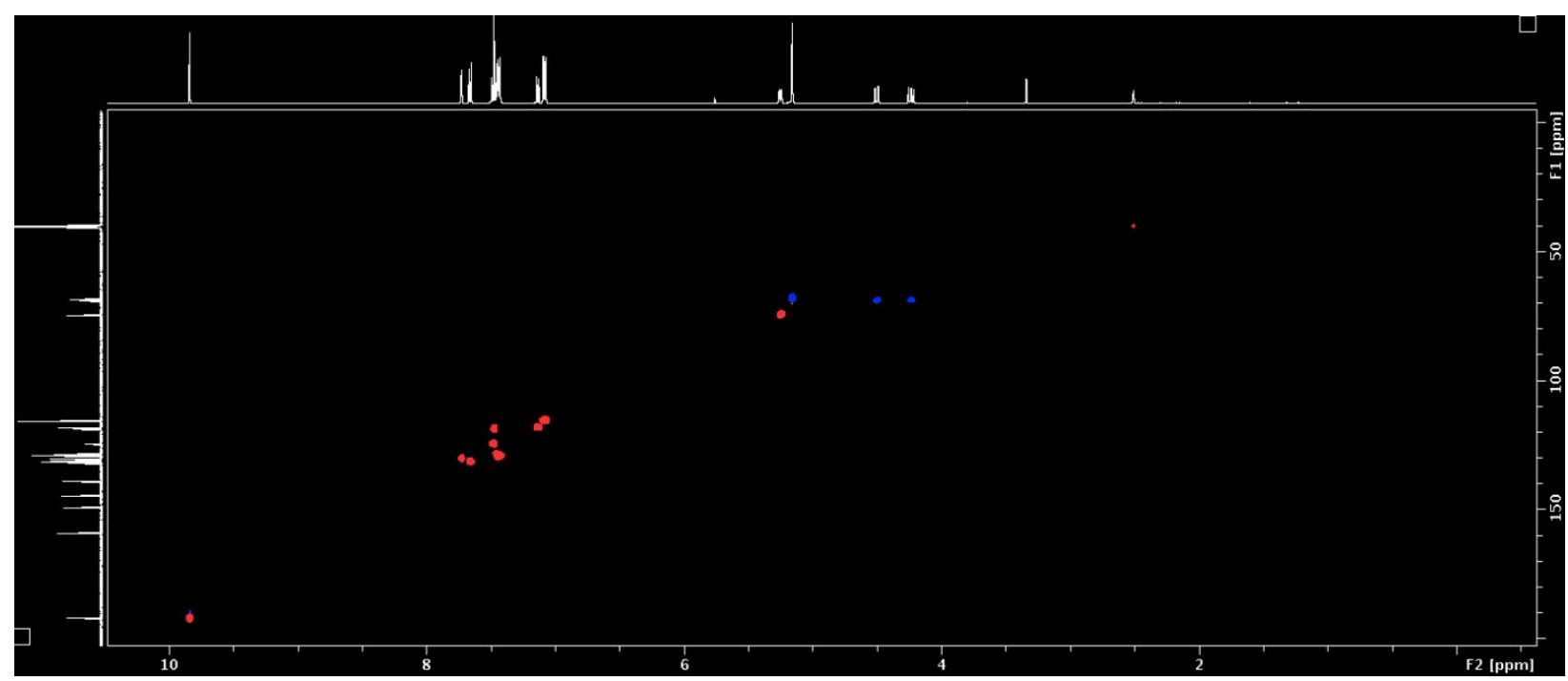

HMBC

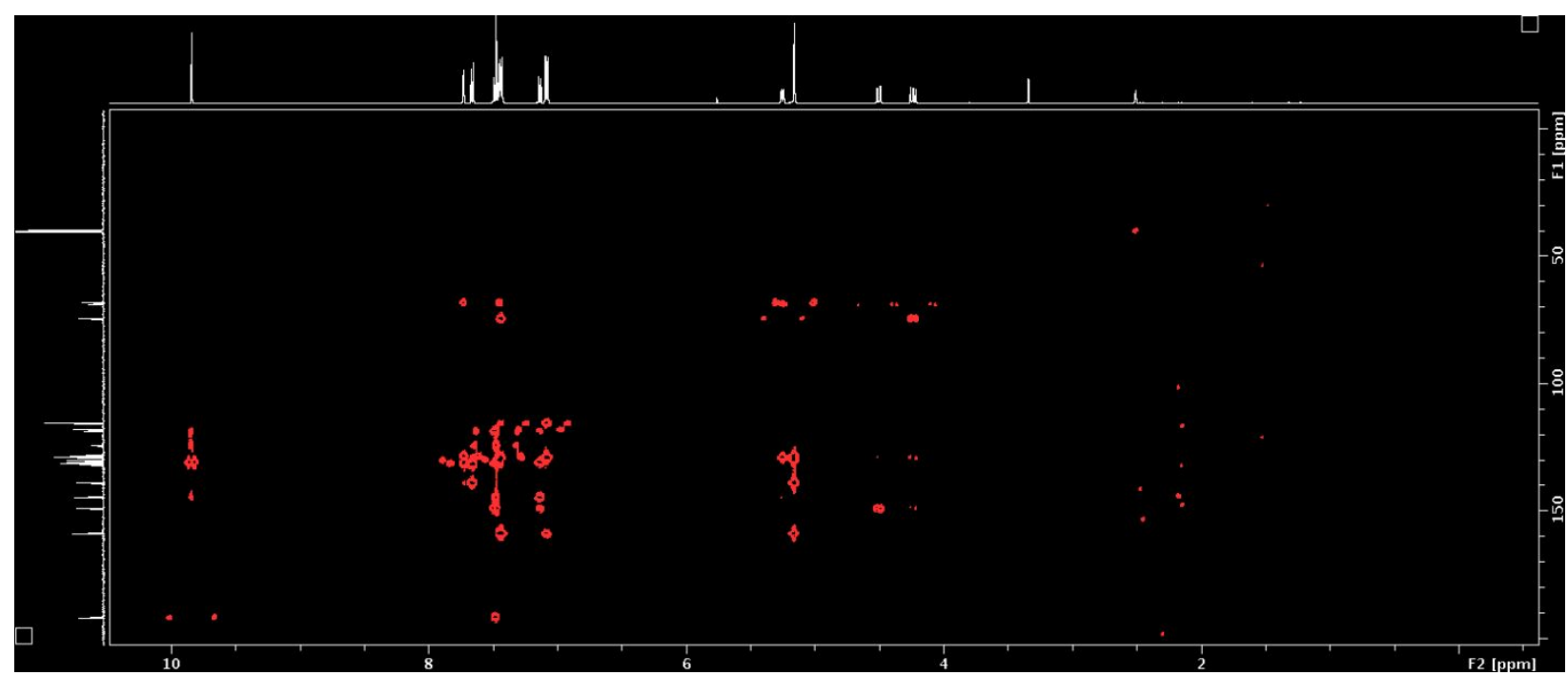


CARBON

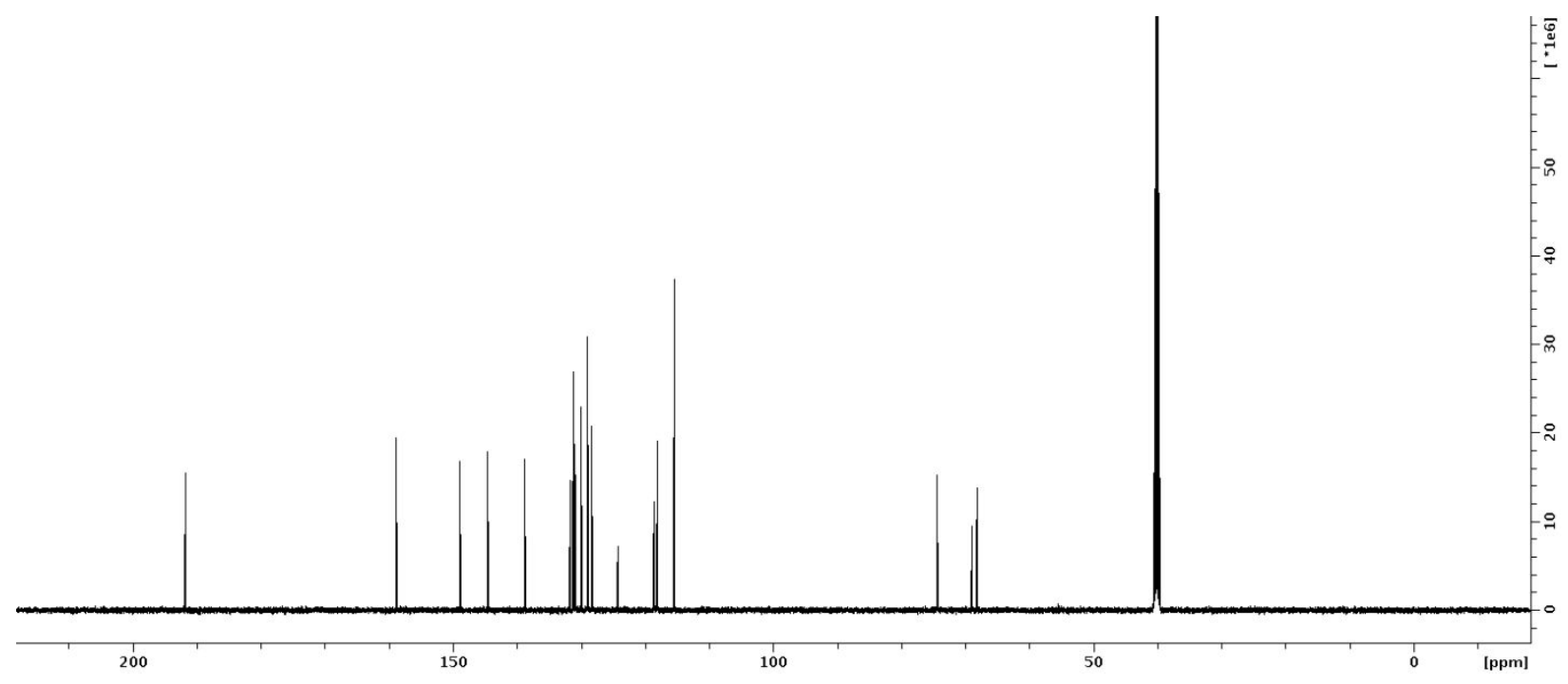

4. Safety Analysis of the high temperature $S_{N} A r$ Cyclization

\section{Conditions}

DSC was completed on a representative reaction mixture for the $S_{N} A r$ cyclization of 2 . The reaction mixture consisted of 2 , in $10 \mathrm{~mL} / \mathrm{mg}$ of diglyme with 3.2 equivalent of DBU. 


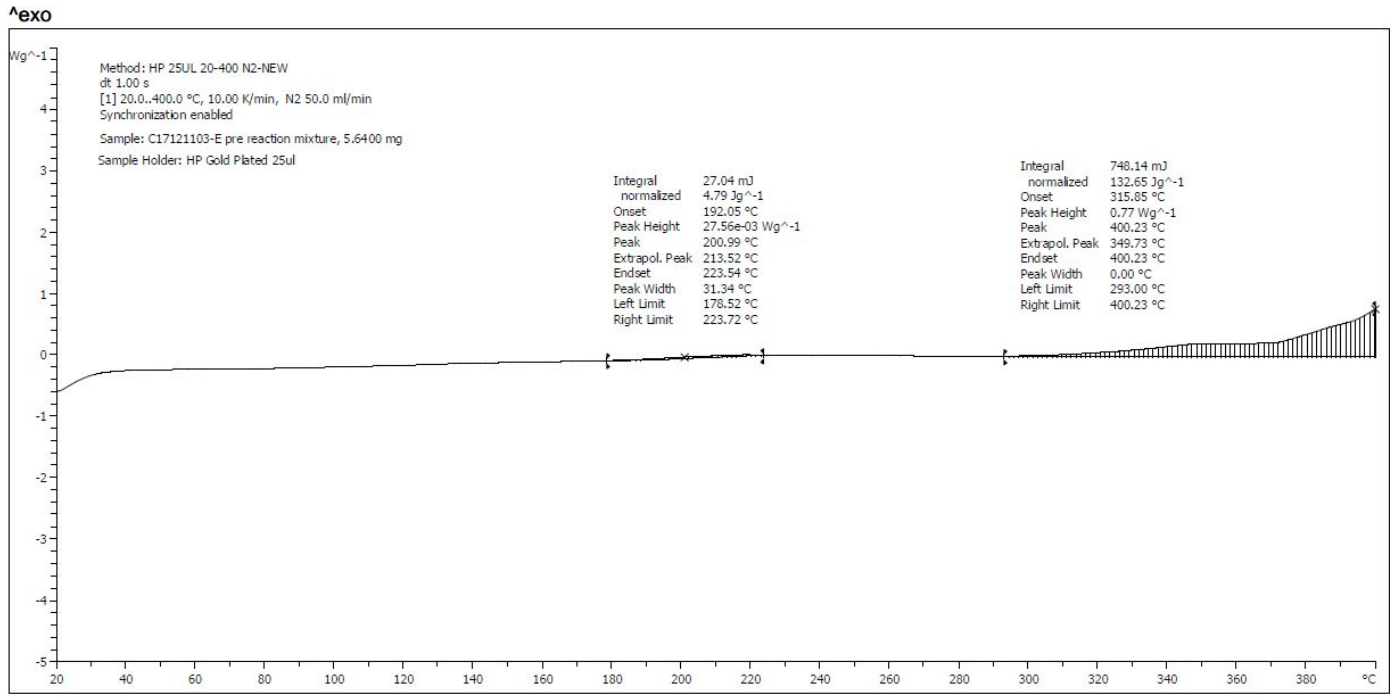

5. Port Connector reactors for Small Scale Screening of High

\section{Temperature Conditions}
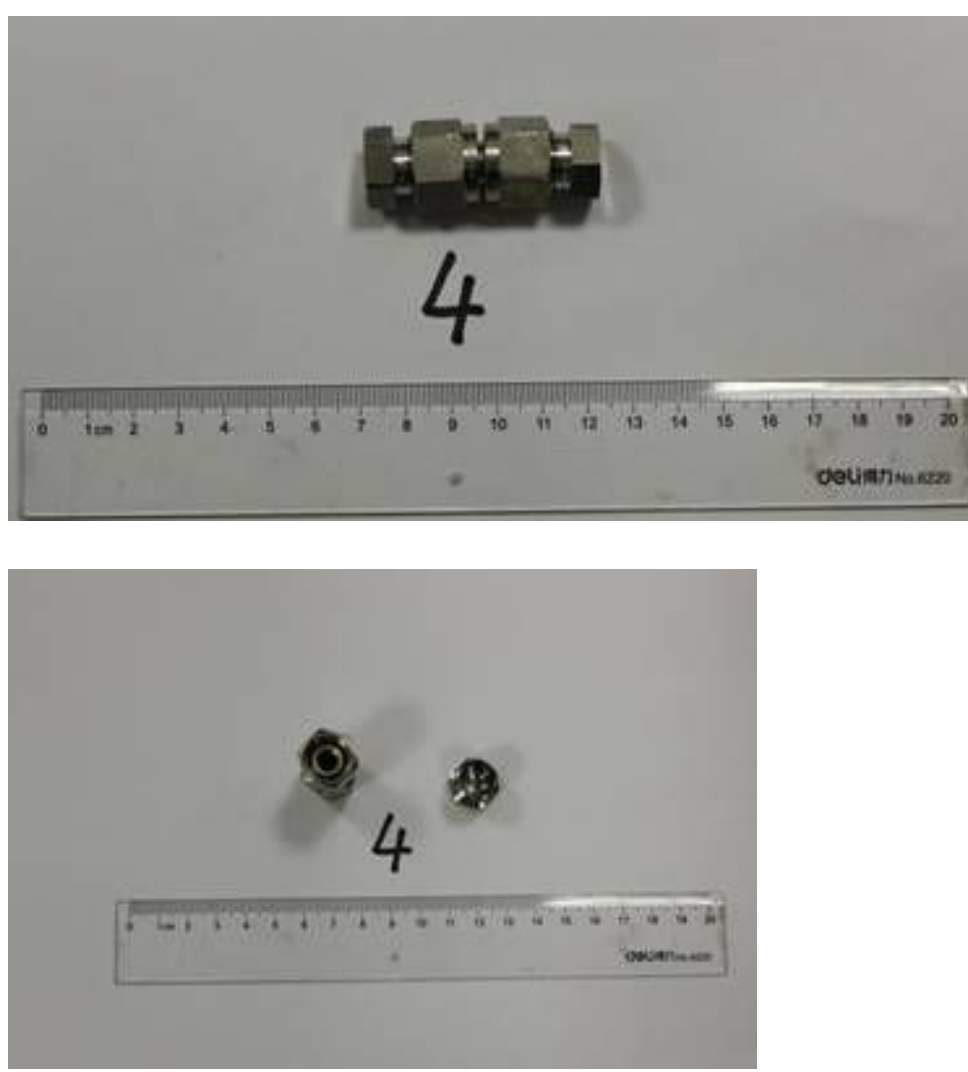
Swagelok port connector reactors are sealed and heated in a GC oven.

These small port connector reactors do not have pressure safety relief. Therefore, an internal safety group in each organization should set safety guidelines based on volume, thermal expansion, \% liquid filled, and potential thermal decomposition to prevent port connector rupture, and shielding for protection in the event of rupture. 